\title{
A comparison of the ability of three common contact lens solutions with different constituents to inhibit growth of Staphylococcus aureus
}

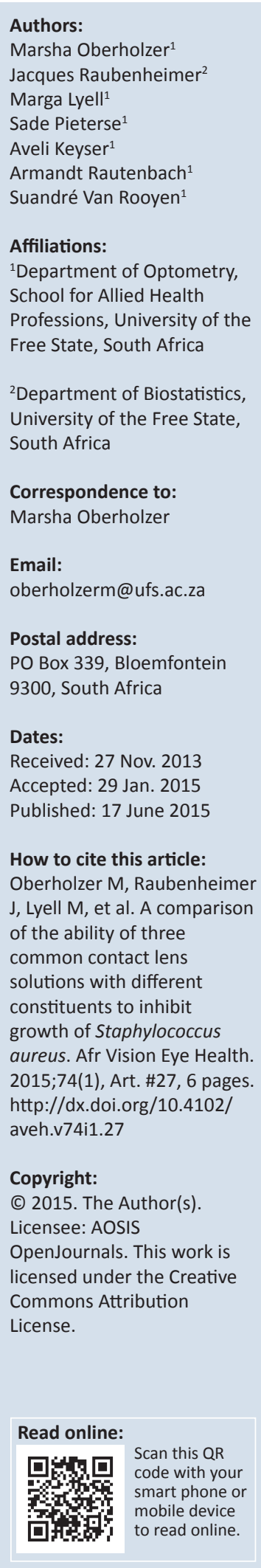

Authors:

Marsha Oberholzer

Marga Lyell ${ }^{1}$

Sade Pieterse

Aveli Keyser

Armandt Rautenbach

ndré Van Rooyen

School for Allied Health

Professions, University of the

University of the Free State,

Correspondence to:

sha Oberholzer

Email:

Postal address:

PO Box 339, Bloemfontein

Dates:

Received: 27 Nov. 2013

Accepted: 29 Jan. 2015

How to cite this article

Oberholzer M, Raubenheimer

J, Lyell M, et al. A comparison

growth of Staphylococcus

aureus. Afr Vision Eye Health.

2015;74(1), Art. \#27, 6 pages.

http://dx.doi.org/10.4102/

Copyright:

(C) 2015. The Author(s)

Licensee: AOSIS

Commons Attribution

License.

\section{Read online:}

code with your

mobile device

to read online.
Background: Staphylococcus aureus is a common commensal on skin and mucosal surfaces; its contact with the eye may cause a variety of ocular inflammations and infections such as blepharitis, conjunctivitis and keratitis, amongst others. Soft contact lenses provide perfect conditions for the breeding of certain pathogens, and disinfecting solutions for contact lenses are therefore of utmost importance. These solutions should be effective in inhibiting the growth of a variety of pathogens to protect the user from ocular infections.

Aim: To highlight the need for clinicians to be aware of the effects of various recommended disinfecting contact lens solutions.

Method: Three popular disinfecting contact lens solutions readily available in South Africa were chosen. These and a control solution (saline) were prepared and inoculated with S. aureus to evaluate the antimicrobial efficacy of each solution. The primary stand-alone test was used to evaluate the solutions according to the ISO standard specifically for this purpose.

Results: The test results indicated that two of the solutions met the ISO standards; the third failed. Of the two that passed the test, only one showed the required 3-log reduction after 30 minutes, as per the ISO standard, although this solution is marketed as a '10 minute system'.

Conclusion: It is important for clinicians to be aware of the complications that may be caused by contaminated solutions, and patients should be warned about the effects thereof. To ensure healthy eyes for our patients, sufficient knowledge regarding the efficacy of recommended multipurpose solutions is necessary. Solutions that meet ISO standards promote good ocular health and ensure sufficient cleaning and disinfecting of contact lenses.

\section{Introduction}

Staphylococcus aureus, a bacterial micro-organism, is a common cause of severe eye infections; it may grow in contact lens solutions that have a reduced ability to inhibit its growth. As clinical practitioners, we expose our patients to contact lens solutions by recommending certain brands for cleaning and disinfecting their contact lenses. As such, we need to be aware of what these solutions contain and their susceptibility to the growth of certain micro-organisms. Clinical practitioners should also educate themselves and be aware of clinical signs and symptoms of contamination of solutions by certain pathogens.

A variety of pathogens, such as S. aureus, Escherichia coli, Pseudomonas aeruginosa and many others may grow in contact lens solutions and, in turn, cause various ocular or corneal inflammations and infections such as corneal ulcers and, in some instances, even enophthalmic conditions. $S$. aureus has been widely recognised as a high-risk pathogen for developing contact lens-related ocular conditions owing to the daily use and abuse of contact lenses..$^{1,2}$

The susceptibility of solutions to the growth of $S$. aureus is therefore of specific importance to contact lens wearers. There is a need to also emphasise that many solutions commercially available today may be susceptible to the growth of a variety of micro-organisms, of which S. aureus has been identified for investigation in the present study. The findings are not intended for marketing purposes or to promote any solution, but rather to create awareness amongst clinicians about a solution's efficacy in eliminating specifically S. aureus.

Three popular solutions currently available in South Africa, and widely used by clinical practitioners, were identified for the study, to compare the ability of each to inhibit the growth of S. aureus. The standardised ISO 14729 primary stand-alone test ${ }^{3}$ was used for testing each solution; the tests were performed at the Microbiology Laboratory of the University of the Free 
State Medical School under the supervision of a medical microbiologist. A solution must meet the ISO standards to be regarded as safe and viable for contact lens wearers. ${ }^{5}$ The present study investigated the logarithmic (log) reductions in S. aureus colonies after disinfection at certain time intervals (10 minutes, 30 minutes, 2 hours, 4 hours and 6 hours) between the 3 test solutions. The results of the study indicated that in these test conditions, 2 of the 3 solutions passed the test after 30 minutes. The manufacturer's usage instructions for one of these solutions, however, indicated sufficient cleaning of contact lenses after 10 minutes. The third solution failed the test.

\section{Literature review}

Contact lens solutions may be described according to the method of working of the specific solution. Of the different solutions currently available commercially, most are described as a 'multiple-purpose solution' (MPS) and are used for the care of soft contact lenses only. These MPSs may be used to rinse and disinfect contact lenses, and to store lenses overnight or when not worn. Typically, these MPSs are composed of a preservative, a buffer and other agents to aid wearing comfort of the lens and also to clean and disinfect lenses. The ability of a MPS to achieve sufficient antimicrobial efficacy is fundamental for the safe use of contact lenses by users. ${ }^{1}$ Effective disinfectant properties of the MPS as well as good lens care compliance by the user reduces the incidence of microbial keratitis by lowering the potential for pathogenic organism growth on both the contact lens surface as well as in the storage case. ${ }^{6}$

To register a contact lens solution as safe and effective against pathogens, and for it to be made available commercially, certain requirements must be met. The standards used are described by Kilvington et al. ${ }^{2}$ and Rosenthal et al. ${ }^{3}$ as the antimicrobial efficacy of the solution as assessed by International Organization for Standardization (ISO) regulation 14729 . The regulation states that a contact lens solution should display appropriate efficacy by either the primary stand-alone test (biocidal) or the secondary regimen test. Kilvington et al. ${ }^{2}$ used two fungi (Fusarium solani and Candida Albicans) and three bacteria (Serratia marcescens, $P$. aeruginosa and S. aureus) as a reference framework for the criteria. Table 1 indicates the criteria set for the efficacy of solutions against these pathogens. ${ }^{7}$ The primary stand-alone test indicates that a tested solution should reduce bacteria colonies by $3 \log _{10}$ units and fungi by $1 \log _{10}$ units, both without regimen. Should a solution not pass the primary stand-alone test, the solution should be re-evaluated according to the regimen qualification in order to be re-evaluated with the secondary regimen test criteria. The regimen qualification criteria and secondary regimen test were not used for the purpose of this research study. However, the criteria are included in Table 1 for completeness of the stated ISO standards.

When a contact lens disinfecting or cleaning solution is purchased, the manufacturer's instructions recommend
TABLE 1: The required average log reduction at disinfection time according to ISO standards.

\begin{tabular}{|c|c|c|c|c|c|}
\hline \multirow[t]{3}{*}{ Criteria } & \multicolumn{5}{|c|}{ Average log reduction at disinfection time } \\
\hline & \multicolumn{2}{|c|}{ Fungi } & \multicolumn{3}{|c|}{ Bacteria } \\
\hline & Fs & $\mathrm{Ca}$ & Sm & $\mathrm{Pa}$ & Sa \\
\hline $\begin{array}{l}\text { Primary criteria of stand- } \\
\text { alone test }\end{array}$ & 1.0 & 1.0 & 3.0 & 3.0 & 3.0 \\
\hline $\begin{array}{l}\text { Secondary criteria of } \\
\text { stand-alone test (regimen } \\
\text { qualification) }\end{array}$ & Stasis & Stasis & $>1$ & $\geq 1$ & $\geq 1$ \\
\hline Regimen criteria & $\leq 10 \mathrm{CFU}$ & $\leq 10 \mathrm{CFU}$ & $\leq 10 \mathrm{CFU}$ & $\leq 10 \mathrm{CFU}$ & $\leq 10 \mathrm{CFU}$ \\
\hline
\end{tabular}

Fs, Fusarium solani; Ca, Candida albicans; Sm, Serratia marcescens; Pa, Pseudomonas aeruginosa; Sa, Staphylococcus aureus.

a specific cleaning regimen to the user to ensure sufficient cleaning and disinfection of the contact lenses. Typically, the instructions specify whether the contact lenses may only be submerged in the solution without rubbing, or whether the contact lenses should be rubbed and rinsed before submersion in the solution. A minimum soaking time of the lenses in the solution is also specified by the manufacturer with which the user should comply to ensure sufficient cleaning and disinfecting of the lenses. Non-compliance has been found to have a profound negative effect on the solution's ability to inhibit organism growth. ${ }^{8,9,10}$

A variety of bacteria may contaminate soft contact lenses, including S. aureus, E. coli and P. aeruginosa, amongst many others. These bacteria may cause a variety of eye infections such as bacterial keratitis and bacterial conjunctivitis, each infection with its own clinical signs and symptoms experienced by the patient. Sakuma et al. ${ }^{7}$ found that more than $50 \%$ of Gram-positive bacteria associated with microbial keratitis are related to the bacteria Staphylococcus epidermidis and S. aureus.

Staphylococcus aureus, a spherical Gram-positive organism, appears as clusters when examined under a microscope and tends to colonise on mucosal surfaces readily and, as a result, also easily infects the eyes. When cultured, S. aureus appears as yellow colonies on agar plates, which assists in distinguishing it from other Staphylococcus spp. This bacterium is responsible for a variety of mild to severe eye infections. According to Kilvington et al. $^{2}$ the cornea is susceptible to infection with this bacterium when the defence system of the cornea is compromised. Typically, a patient with bacterial keratitis resulting from a contact lens solution with inferior disinfecting ability will present with ocular symptoms characterised by severe eye discomfort. Presentation is typically unilateral, associated with acute pain and hyperaemia as well as photophobia. Vision in the affected eye may also be compromised. Expected clinical signs include focal stromal infiltrates on the cornea with an overlying area of epithelial excavation, resulting in a lesion with time and a mucopurulent discharge. In severe cases, a pronounced anterior chamber reaction (cells and flare) and hypopyon may present additionally. ${ }^{1}$

Some comparative studies have been done in the last decade or more, investigating the disinfecting abilities of a variety of MPS solutions to $S$. aureus, amongst other pathogens. 
Rosenthal et al. ${ }^{6}$ conducted a study where OPTI-FREE Express with Aldox was compared with several other contact lens disinfecting solutions. OPTI-FREE Express with Aldox is preserved with polyquaternium-1 and myristamidopropyldimethylamine, whereas the compared solutions included 3\% hydrogen peroxide systems and MPS preserved with polyhexamethylenebiguanide. These products were exposed to more testing for antimicrobial activity against $S$. aureus. The results of the study showed that OPTI-FREE Express with Aldox provided a broader range of antimicrobial activity than the other MPSs; however, the activity was comparable to that of the $3 \%$ hydrogen peroxide systems but regrowth was less during extended storage than the $3 \%$ hydrogen peroxide systems.

A recent study by Mohammadinia et al. ${ }^{5}$ evaluated the antibacterial efficacy of three contact lens disinfecting solutions: ReNu MultiPlus, Solo Care Aqua and All-Clean Soft. All three solutions were introduced with standard strains of $P$. aeruginosa and S. aureus. Samples of the solutions were evaluated according to the survival of micro-organisms at the manufacturer's recommended minimum time for disinfection. All three solutions evaluated in the study showed a greater than required mean $3 \log _{10}$ unit reduction against the named strands of bacteria, and consequently the antibacterial effectiveness of the solutions was regarded as acceptable based on the ISO 14729 primary stand-alone test criteria. Of the three solutions, however, ReNu MultiPlus showed less reduction of the $S$. aureus clinical isolate than the other two solutions evaluated.

Sakuma et al. ${ }^{7}$ compared the efficacy of four soft contact lens solutions ( $\mathrm{ReNu}$, Complete, Opti-One and Opti-Free) against the organisms S. epidermidis and P. aeruginosa. The results of the study indicated that the amount of microbes eliminated over 4 hours ranged from $0.5 \log _{10}$ units $(\mathrm{ReNu})$ to $2.5 \log _{10}$ units (Complete). Opti-One and Opti-Free eliminated the entire inoculum of $S$. epidermidis in 10 minutes, whereas neither Complete nor ReNu completely eliminated the microbes after 4 hours. ReNu and Complete reduced the microbe count by 3.0 and $3.5 \log _{10}$ units, respectively. Lever and Roya ${ }^{4}$ set out to determine the antimicrobial efficacy of 10 commercially available MPSs on the European market. The efficacy of the solutions was also rated according to the ISO primary stand-alone test. The results of this study indicated that ReNu MultiPlus, ReNu Multi-Purpose, Complete and DUA MPS meet the ISO primary stand-alone test acceptance criteria for stand-alone disinfectants against all challenge organisms: S. aureus, $S$. marcescens, $P$. aeruginosa, C. albicans and F. solani. However, Solo Care Soft, All-In-One Light, Optiplus, Opti-Free Express, UniCare and Combi Comfort MPS did not meet the ISO primary stand-alone test criteria for one or more test organisms within their respective labelled minimum disinfection times. Only ReNu MultiPlus and ReNu Multi-Purpose solutions additionally exceeded the minimum ISO primary stand-alone test acceptance criteria within $25 \%$ (one hour) of their labelled minimum disinfection time for all test organisms.
Further studies have also compared the effectivity of solutions in inhibiting the growth of organisms other than S. aureus. ${ }^{11,12}$

In the present study, the authors investigated the efficacy of three commercially available MPSs in South Africa against the pathogen $S$. aureus. The three solutions were Solo Care, ReNu MPS and Opti-Free Express MPS.

\section{Methodology}

The study was of a prospective experimental, beforeand-after comparative nature. Three solutions commonly available in South Africa, and often recommended by optometrists, were chosen for evaluation; these were Solo Care, ReNu MPS and Opti-Free Express MPS. The manufacturers' recommended times for sufficient cleaning and disinfection of contact lenses were stated in the usage instructions as 6 hours (Opti-Free Express, solution A), 10 minutes (ReNu MPS, solution B) and 4 hours (Solo Care, solution $\mathrm{C}$ ). The antimicrobial efficacy of the regimen was evaluated according to the ISO standards required for the primary stand-alone test which only requires 'stand-alone' of the MPS. The manufacturer's instructions for ReNu MPS, however, also recommended rubbing of the contact lens for better cleaning, and compliance with the cleaning regimen has been shown to be a critical factor in inhibiting organism growth; $;, 9,10,13$ however, as the aim of the study was to evaluate the disinfecting ability of each of the MPSs and as no contact lenses were involved in the experimental process, this step was not foreseen as a problem. The other two MPSs used in the study, Opti-Free Express and Solo Care, recommended only soaking of the lenses with no rubbing for sufficient cleaning and disinfection. The criteria recommended for the MPSs therefore mostly corresponded with the criteria in the ISO primary stand-alone test. ${ }^{2}$

The standardised ISO 14729 primary stand-alone test was used for testing each MPS; tests were conducted in the clinical Microbiology Laboratory of the University of the Free State Medical School. All tests were performed under the supervision of a medical microbiologist. The primary stand-alone test used in the present study only involves the evaluation of the chosen MPS, and accordingly no contact lenses were involved in the experimental procedure. The primary stand-alone test has been specifically developed to evaluate the ability of a solution to inhibit the growth of a specified bacterium. If a MPS should pass the primary stand-alone test according to the criteria previously stated, there would be no need to perform the second test, known as the secondary regimen test. However, if the MPS fails the primary stand-alone test, the evaluation process should be repeated with the secondary regimen test. The secondary regimen test should then be passed for a solution to adhere to the ISO standard and therefore be regarded as safe for use by the public. For the requirements of this study, only the primary stand-alone test was performed. It should therefore be kept in mind that, should a MPS fail the initial criteria of the primary stand-alone test, the MPS should not be regarded 
as unsafe, as the MPS should then be subjected to the secondary regimen test, which was not used in the present study.

A total of 40 test tubes were used for the evaluation of the 3 solutions plus a control solution (sterile saline). For each time interval tested (10 minutes, 30 minutes, and 2, 4 and 6 hours) two test tubes were prepared for each of the 4 solutions. The total of 40 test tubes were marked as Solution A, B, C and control to prevent bias. The identity of each of the solutions named A, B and C was only familiar to the medical microbiologist assisting in the study and was only made known to the researchers after completion of the study.

The inoculum was prepared according to the ISO 14729 primary stand-alone test criteria which requires between $1 \times 10^{5}$ and $1 \times 10^{6}$ colony-forming units per $\mathrm{mL}(\mathrm{CFU} / \mathrm{mL})$. As midpoint, $5 \times 10^{5} \mathrm{CFU} / \mathrm{mL}$ was chosen, and a suspension with a $0.5 \mathrm{McF}$ arland standard for S. aureus $\left(1.5 \times 10^{8} \mathrm{CFU} / \mathrm{mL}\right)$ was used for the preparation of the inoculum, as advised by the microbiologist. The inoculum was prepared with $1 \mathrm{~mL}$ of the $0.5 \mathrm{McF}$ arland standard preparation $\left(1.5 \times 10^{8} \mathrm{CFU} / \mathrm{mL}\right)$ and was added to $2 \mathrm{~mL}$ of sterile saline/BHI broth (dilution ratio 1:3). This ratio provided the researchers with $0.5 \times 10^{8}$ $\mathrm{CFU} / \mathrm{mL}$ after which $1 \mathrm{~mL}$ of the above preparation was again mixed with $9 \mathrm{~mL}$ of sterile saline (dilution ratio: 1:10). The final inoculum was therefore $5 \times 10^{5} \mathrm{CFU} / \mathrm{mL}$ (500 000). The final prepared suspension in the test tubes was $2 \mathrm{~mL}$ and consisted of $1900 \mu \mathrm{L}$ of solution A, B, C or the control solution and $100 \mu \mathrm{L}$ of the inoculum.

To confirm sterility of the suspension, an uninoculated specimen of the various solutions was plated on agar plates to determine whether any micro-organisms were present in any of the solutions. A control measurement using the sterile saline solution was used to obtain a mean of uninhibited growth for comparison with the other 3 solutions. A single measurement of each solution was regarded as insufficient for the measurement of averages and therefore repeated measurements ( 2 per solution, per time interval) provided averages.

After successful preparation of the inoculum in the test tubes, a single standard suspension of the $S$. aureus bacterium was made using the ATCC strain in the laboratory and inserted in each test tube. The suspensions were left to incubate at room temperature $\left(35^{\circ} \mathrm{C}-37^{\circ} \mathrm{C}\right)$. After each of the specified time intervals, $100 \mu \mathrm{L}$ of each of the prepared inoculums were plated onto blood agar plates and incubated overnight at $35{ }^{\circ} \mathrm{C}$ in ambient air. This was followed by colony counts and the calculation of a mean for each suspension per time interval. The equation used to determine the log reduction in growth of the S. aureus in each solution and the control, was: ${ }^{5}$

Log reduction $=\log _{10}($ initial $\mathrm{CFU} / \mathrm{mL})$

$$
-\log _{10}(\text { final CFU /mL) }
$$

\section{Results}

Table 2 shows the actual CFU count as the average of the reading across both plates. It should be noted that for both $\mathrm{ReNu}$ and Solo Care, no CFUs were observed after 30 minutes. It can also be seen that for the saline solution, the growth increased to a peak, after which a slow decline was seen.

Table 3 indicates the log reduction of each solution at each of the stated time intervals. Figure 1 is a graphical presentation of the data in Table 2.

Putting Figure 1 into perspective, the control solution showed increases from zero to $16.8 \%$ and then $183.2 \%$ at 10 minutes and 30 minutes respectively. Both ReNu and Solo Care had reduced the CFU count by more than $99 \%$ at 10 minutes, and by $100 \%$ at 30 minutes, while Opti-Free reduced the CFU count by $38.4 \%$ and $83.6 \%$ at those two respective times. By 6 hours, Opti-Free had reduced the CFU count by $94.4 \%$.

It can be seen in Table 3 that Opti-Free Express (Solution A) failed the ISO primary stand-alone test at each of the indicated time intervals. The failure of the primary stand-alone test makes the solution subject to the secondary regimen test for meeting

TABLE 2: Actual colony counts per solution per time interval.

\begin{tabular}{lcccc}
\hline Time & $\begin{array}{c}\text { Solution A: Opti-Free } \\
\text { Express }\end{array}$ & $\begin{array}{c}\text { Solution B: } \\
\text { ReNu }\end{array}$ & $\begin{array}{c}\text { Solution C: } \\
\text { Solo Care }\end{array}$ & $\begin{array}{c}\text { Control: } \\
\text { Saline }\end{array}$ \\
\hline Zero & 25000 & 25000 & 25000 & 25000 \\
10 minutes & 15400 & 100 & 200 & 29200 \\
\hline 30 minutes & 4100 & 0 & 0 & 70800 \\
Two hours & 5000 & 0 & 0 & 54100 \\
Four hours & 2400 & 0 & 0 & 49000 \\
Six hours & 1400 & 0 & 0 & 32200 \\
\hline
\end{tabular}

TABLE 3: Log reduction per solution per time interval.

\begin{tabular}{lcccc}
\hline Time & $\begin{array}{c}\text { Solution A: Opti-Free } \\
\text { Express }\end{array}$ & $\begin{array}{c}\text { Solution B: } \\
\text { ReNu }\end{array}$ & $\begin{array}{c}\text { Solution C: } \\
\text { Solo Care }\end{array}$ & $\begin{array}{c}\text { Control: } \\
\text { Saline }\end{array}$ \\
\hline 10 minutes & 0.210 & 2.398 & 2.097 & -0.067 \\
30 minutes & 0.785 & 4.398 & 4.398 & -0.452 \\
Two hours & 0.699 & 4.398 & 4.398 & -0.335 \\
Four hours & 1.018 & 4.398 & 4.398 & -0.292 \\
Six hours & 1.252 & 4.398 & 4.398 & -0.110 \\
\hline
\end{tabular}

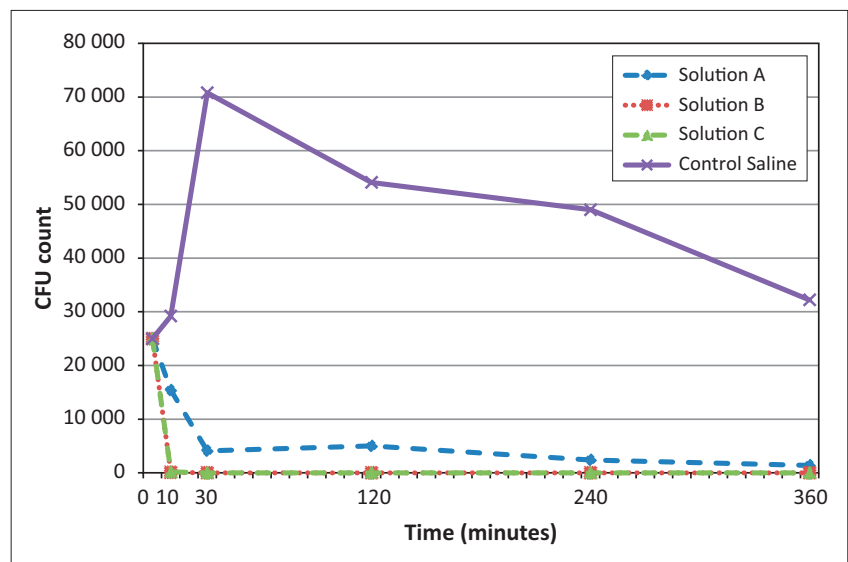

CFU, colony-forming units.

FIGURE 1: Actual colony counts per solution per time interval presented graphically. 
ISO standards. The solution showed a maximum log reduction of 1.252 after 6 hours and, as stated before, the minimum requirement to pass the primary stand-alone test, is a 3-log reduction of $S$. aureus. The recommended disinfection time of Opti-Free Express stated in the manufacturer's instructions is 6 hours; however, after 6 hours, the solution did not show the required 3-log reduction and should be re-evaluated with the secondary regimen test that allows the lens care regimen to be implemented with actions such as rubbing and rinsing of the contact lens as part of the cleaning regimen. Opti-Free Express will consequently have to pass the second test according to ISO 14729 to prevent the contact lens from being susceptible to causing ocular infections such as microbial keratitis.

ReNu MPS (Solution B) passed the ISO primary stand-alone test after 30 minutes; however, the manufacturer's instructions recommend 10 minutes' disinfection time, and sufficient disinfection of the bacteria was only noted after 30 minutes. The manufacturer's instructions also recommend rubbing the contact lens for better cleaning. Owing to the nature of the primary stand-alone test and no contact lenses being involved in the experimental process, it must be kept in mind that there is a possibility that the action of the solution may be improved with sufficient disinfection in 10 minutes with rubbing of the contact lens in this solution. ReNu MPS therefore passed the primary stand-alone test after 30 minutes, and subjecting the solution to the second test was not indicated.

Solo Care (Solution C) passed the ISO primary standalone test after 30 minutes and already showed a $2.097 \mathrm{log}$ reduction after 10 minutes. The manufacturer's instructions state correctly that 4 hours of soaking (without rubbing of the lens) in the solution is recommended for sufficient disinfection of a contact lens. The cleaning recommendations from the manufacturer are accordingly correct and this solution too need not be re-evaluated with the second test.

According to the ISO primary stand-alone test, Solo Care and ReNu MPS showed similar antimicrobial activity against S. aureus, followed by Opti-Free Express.

\section{Discussion}

Optometric patients wearing contact lenses are exposed daily to a higher risk of developing ocular infections, especially when the recommended cleaning regimen of their lenses is not followed adequately. The responsibility to warn patients of the warning signs and symptoms of ocular infections related to contact lens wear therefore lies with the clinician. Contact lenses, especially the soft variety, may provide the perfect breeding circumstances for pathogens such as $S$. aureus and good cleaning regimens and superior disinfecting contact lens solutions are essential to guarantee the health of the patient's eyes.

Previous studies similar to the present study have been conducted with variable results. The findings of the present study support the findings of Lever and Roya ${ }^{4}$ who indicated that Opti-Free MPS failed the primary stand-alone test criteria. Mohammadinia et al. ${ }^{5}$ also had similar findings in their study, where both $\mathrm{ReNu}$ and Solo Care MPS passed the ISO standards of the primary stand-alone test. The findings of the present study, however, do not agree with studies by Rosenthal et al. ${ }^{6}$ and Sakuma et al., ${ }^{7}$ who found that Opti-Free MPS passed the ISO standards of the primary stand-alone test. In the article by Sakuma et al., ${ }^{7} \mathrm{ReNu}$ MPS failed the primary stand-alone criteria. However, the study by Lever and Roya ${ }^{4}$ again passed the ReNu MPS according to ISO standards and failed Solo Care MPS as the latter solution could not show a 3-log reduction to meet the primary stand-alone test criteria. None of the abovementioned studies proceeded to test the failed solutions with the secondary regimen test. The above-mentioned studies were all conducted in different environments and different laboratories. The time intervals according to which the studies were done are not clear and, consequently, varying environments and circumstances might have contributed to the variability of the results. Campbell et al. ${ }^{14}$ conducted a study in which Opti-Free and ReNu were tested blindly by a large number of contact lens wearers over a period of 90 days (i.e. participants also followed the approved cleaning regimen). They found that Opti-Free was rated better in terms of subjective experience by the participants, and there were no significantly different adverse events, confirming that, in conjunction with compliance with the correct regimen, Opti-Free may well be suited for general public use.

The results of the study showed that two of the three solutions passed the primary stand-alone test after 30 minutes; however, one of these solutions had a recommended disinfection time in the manufacturer's instructions of only 10 minutes, whereas the other solution correctly recommended 4 hours for disinfection.

Table 3 and Figure 1 both present supportive evidence that Opti-Free Express did not show a 3-log reduction within 6 hours, and this solution should be re-evaluated with the secondary regimen test. ReNu and Solo Care MPS showed sufficient disinfecting abilities to meet the primary standalone test criteria; however, the manufacturer's instructions for $\mathrm{ReNu}$ MPS should be reviewed as this solution is marketed as a 10-minute system and only showed a 3-log reduction after 30 minutes had elapsed. Conversely, it is marketed as a 'rub solution', which may also be the cause for the slightly delayed working of the solution and it may be assumed that, with rubbing of a contact lens, the working of the solution may meet the required 3-log reduction within 10 minutes as stated in the instructions.

It is important for us as clinicians to be aware of the effects of contaminated solutions. It is also important to note that our patients should be motivated at every visit and eye exam to follow the provided and recommended cleaning regimen to ensure reduced risk of ocular infections. Educating our patients on the effects and ocular signs and symptoms of eye infections as a result of contact lens use and abuse is important to enable them to recognise the warning signs at an early stage and seek help. 


\section{Conclusion}

Soft contact lenses may easily be contaminated with various pathogens such as $S$. aureus and, as a result, cause mild to severe eye infections. The efficacy of a contact lens MPS to inhibit the growth of pathogens such as S. aureus on lens surfaces and in storage cases is therefore of utmost importance. ISO standards for MPSs have been developed to ensure that solutions available to the public are safe and provide adequate cleaning and disinfection of contact lenses. Three MPSs currently available on the South African market were subjected to the primary stand-alone test to investigate whether they meet ISO standards. One of the three solutions failed the primary stand-alone test criteria, but might meet ISO standards when re-evaluated with the secondary regimen test which, however, was not conducted for the purpose of the present study.

Our findings emphasise the need for education of both clinicians as well as patients/users on the risks of ocular eye infections possibly caused by contaminated solutions and insufficient cleaning and disinfection of contact lenses. More awareness should be created about recommended cleaning regimens to ensure sufficient cleaning and disinfection of contact lenses and close monitoring of the product to ensure compliance with the manufacturers' claims. It is also recommended that manufacturers review their recommended instructions for sufficient disinfection of contact lenses and that solutions should be frequently reviewed according to the ISO standards. These standards serve as a tool to ensure low risk of ocular infections in our contact lens-wearing patients and the re-evaluation of MPSs according to these standards is necessary. It should be emphasised that clinicians should recommend to their patients MPSs that meet ISO standards and offer sufficient disinfection of contact lenses to reduce the risks of ocular infections and in turn protect both the patient as well as the clinician.

\section{Acknowledgements}

We thank all the learners who willingly participated in the study, and Dr Justyna Wojno, pathologist (NHLS), Faculty of Health Sciences, University of the Free State, for assistance with the laboratory tests.

\section{Competing interests}

The authors declare that they have no financial or personal relationships which may have inappropriately influenced them in writing this article.

\section{Authors' contributions}

M.O. (University of the Free State) was the project leader. The student group consisted of M.L. (University of the Free State), S.P. (University of the Free State), A.K. (University of the Free State), A.R. (University of the Free State) and S.v.R. (University of the Free State), final year Optometry students at the University of the Free State. The students (M.L, S.P, A.K, A.R. and S.v.R) were responsible for the experimental and project design. They also conducted all of the microbiological experiments, preparation of samples and needed calculations with the help of a medical microbiologist (as mentioned in acknowledgments). M.O. oversaw the protocol and study. J.R. was responsible for the statistical analyses of the data for the study.

\section{References}

1. Kanski JJ, Bowling B. Clinical ophthalmology: A systematic approach. 7th edn London: Elsevier; 2011.

2. Kilvington $\mathrm{S}$, Huang $\mathrm{L}$, Kao $\mathrm{E}$, Powell $\mathrm{CH}$. Development of a new contact lens multipurpose solution: Comparative analysis of microbiological, biological and clinical performance. J Optom. 2010;3:134-142. http://dx.doi.org/10.1016/ S1888-4296(10)70019-4

3. Rosenthal RA, Sutton SVW, Schlech BA. Review of standard for evaluating the effectiveness of contact lens disinfetants. PDA J Pharm Sci Technol. 2002;56:37-50

4. Lever AM, Borazjanit RN. Comparative antimicrobial efficacy of multi-purpose hydrogel lens care solutions. Cont Lens Anterior Eye. 2001;24:94-99. http:// dx.doi.org/10.1016/S1367-0484(01)80023-0

5. Mohammadinia M, Rahmani S, Eslami G, et al. Contact lens disinfecting solutions antibacterial efficacy: Comparison between clinical isolates and the standard 2012;26:327-330. http://dx.doi.org/10.1038/eye.2011.284

6. Rosenthal RA, Bell WM, Abshire R. Disinfecting action of a new multi-purpose disinfection solution for contact lenses. Cont Lens Anterior Eye. 1999;22:104-109. http://dx.doi.org/10.1016/S1367-0484(99)80010-1

7. Sakuma S, Reeh B, Dang D, Harris MG. Comparative efficacies of four soft contact lens disinfection solutions. Int Contact Lens Clin. 1996;23:234-241. http://dx.doi. org/10.1016/S0892-8967(96)00108-3

8. Zhu H, Bandara MB, Vijay AK, Masoudi S, Wu D, Willcox MDP. Importance of rub and rinse in use of multipurpose contact lens solution Hua. Optom Vis Sci. 2011;88:967-972. http://dx.doi.org/10.1097/OPX.0b013e31821bf976

9. Rosenthal RA, Henry $\mathrm{CL}$, Stone RP, Schlech BA. Anatomy of a regimen: Consideration of multipurpose solutions during non-compliant use. Cont Lens Anterior Eye. 2003;26:17-26. http://dx.doi.org/10.1016/S1367-0484(02) 00085-1

10. Kilvington S, Lonnen J. A comparison of regimen methods for the removal and inactivation of bacteria, fungi and Acanthamoeba from two types of silicone hydrogel lenses. Cont Lens Anterior Eye. 2009;32:73-77. http://dx.doi. org/10.1016/j.clae.2008.12.008

11. Wilson L, Sawant A, Ahearn D. Comparative efficacies of soft contact lens disinfectant solutions against microbial films in lens cases. Arch Ophthalmol. 1991;109:11551157. http://dx.doi.org/10.1001/archopht.1991.01080080115043

12. Santos L, Oliveira R, Oliveira MECDR, Azeredo J. Lens material and formulation of multipurpose solutions affects contact lens disinfection. Cont Lens Anterior Eye. 2011;34:179-182. http://dx.doi.org/10.1016/j.clae.2011.02.002

13. Kilvington S, Powell CH, Lam A, Lonnen J. Antimicrobial efficacy of multi-purpose contact lens disinfectant solutions following evaporation. Cont Lens Anterior Eye. 2011;34:183-187. http://dx.doi.org/10.1016/j.clae.2011.02.010

14. Campbell R, Kame G, Leach N, Paul M, White E, Zigler L. Clinical benefits of a new multipurpose disinfecting solution in silicone hydrogel and soft contact lens users. Eye Contact Lens. 2012;38:93-101. http://dx.doi.org/10.1097/ ICL.0b013e318243c1a3 